\title{
Preventing the introduction of Ebola virus into the Eastern Mediterranean Region: enhanced preparedness is the key
}

\author{
Mamunur Rahman Malik, Jaouad Mahjour ${ }^{2}$ and Ala Alwan ${ }^{3}$
}

The current outbreak of Ebola virus disease (EVD) in West Africa is unlike any of the previous outbreaks caused by the disease because of the unprecedented speed with which it is evolving. A number of countries in western Africa are currently affected - Guinea, Liberia, Sierra Leone and Democratic Republic of Congo. All these countries have now reported laboratory-confirmed cases. The outbreak, which probably began as an event of acute febrile illness associated with a high case fatality rate, was first identified in December 2013 in the Guéckédou region of GuineaConakry, a remote region of southeast Guinea bordering Sierra Leone and Liberia (1). It soon escalated with rapidly increasing numbers of cases reported from these three countries, possibly due to constant movement of people across their porous borders, raising a high public health alert across the continent. The World Health Organization was officially notified of an outbreak of EVD on 23 March 2014 (2).

The exponential increase in cases from this EVD outbreak and its unprecedented size, pace and scale led WHO on 8 August 2014 to declare this event a public health emergency of international concern (PHEIC) under the International Health Regulations (2005) (3). More recent incidents include the travel-associated spread of EVD to Nigeria and Senegal and the identification of Ebola transmission in the Democratic Republic of Congo, although the molecular data suggest that this event is unrelated to the ongoing outbreak in West Africa (4).

Being a zoonotic pathogen, the circulation of Ebola virus among humans is uncommon. Transmission of Ebola virus to humans probably originates from an animal reservoir, possibly involving additional intermediary species, and occurs through direct contact with tissue or bodily fluids of an infected animal (5). This explains the intermittent and unpredictable nature of outbreaks of EVD. In most previous outbreaks, the virus emerged in geographically restricted, rural regions and the outbreaks were contained through routine public health measures such as case identification, contact tracing, patient isolation and quarantine to break the chain of virus transmission. None had spread beyond East and Central Africa (6).

The current epidemic is not only by far the largest and most persistent outbreak of the disease reported in its 38-year history, it is also the first ever multi-country outbreak $(2,6)$. After recording cases for almost 10 months, there have been 8033 cases of EVD (confirmed, probable, and suspected) and more than 3879 associated deaths reported from this outbreak as of 8 October 2014 (7); this is more than in all previous Ebola outbreaks combined. What's more, these numbers are likely to be underestimates, given the limitations of case identification and possible weakness in contact tracing and laboratory identification. In a recent report, a conservative projection has been suggested of close to 20000 cases by early November 2014 (2), an ominous sign that the epidemic is continuing to expand.

\section{Threat of importation of EVD in the Eastern Mediterranean Region}

Paradoxically Ebola is not new to the Region. The first outbreak of EVD was identified in Sudan in 1976, simultaneously (8) with Democratic Republic of Congo, in a remote village which is currently part of South Sudan. Since then Sudan faced two more outbreaks of EVD, in 1979 and 2004, in places which are now part of South Sudan (9). Still, local human Ebola transmission has never occurred outside Africa in the history of this disease. However, it has been projected recently that as long as the transmission remains uncontrolled at the epicentre of the outbreak, Ebola could become endemic in West Africa; it could then become a reservoir for the virus's spread to other parts of Africa and beyond (10). The recent evidence of the occurrence of travel-associated cases of EVD in Nigeria and Senegal strongly supports this assumption and therefore the likelihood of EVD spreading to other countries, including to the

${ }^{\top}$ Regional Adviser, Pandemic and Epidemic Diseases, Department of Communicable Diseases, World Health Organization Regional Office for the Eastern Mediterranean, Cairo Egypt(malikm@who.int).

${ }^{2}$ Director, Department of Communicable Diseases, World Health Organization Regional Office for the Eastern Mediterranean, Cairo Egypt.

${ }^{3}$ Regional Director, World Health Organization Regional Office for the Eastern Mediterranean, Cairo Egypt. 
Eastern Mediterranean Region, should not be taken lightly. In fact, based on air traffic connections between West African countries and those in the Region, some modelling data have already projected that the risk of importation of EVD cases to some of the countries in the Region is moderately high (11).

A number of countries in the Region have international travel hubs that connect them (by daily/weekly flights) with those of western Africa where the outbreak is currently ongoing. In all likelihood, transmission of Ebola through international travel would be the most probable scenario for the introduction of EVD in countries of the Region. Due to the long incubation period of EVD, which ranges from 2 to 21 days (5), it is possible that a person incubating the disease might fly to any country in the Region seemingly healthy and be allowed entry without suspicion. Furthermore, at a later stage once a person becomes ill and presents to a health facility, the symptoms of Ebola may be masked by other diseases as the earlier clinical symptoms of EVD often resemble other infections like malaria, typhoid, Lassa fever, hepatitis, yellow fever or even influenza (12). The risk of transmission by land or sea is conceivable but far less likely while it is presumed that the importation of exotic animals (monkeys and other primates who are the intermediate host of the virus) also carries risk (13).

Given the threat of importation of EVD into the Region, national and regional efforts need to be urgently stepped up in order to detect early any possible introduction of Ebola virus into any of the countries and to enhance readiness for prompt response so as to limit local transmission and subsequent spread of the virus. A number of key public health actions (Box 1) should be considered by the countries in line with WHO's recommended approach to accelerate national preparedness efforts at all levels of health systems, including at points of entry (14).

\section{Challenges of early recognition}

While the probability that Ebola virus establishes itself in the Region may be low, given the geographic expansion of the outbreak in West Africa and in the absence of any established foci for Ebola virus in the Region, the threat of introduction is real. Therefore the gravity of the situation requires countries to give serious consideration to preparedness.

Recognizing the signs of EVD can be challenging for countries of the Region as Ebola is unknown here and the surveillance systems may not pick up a case early because of different possible scenarios of introduction of the virus into the Region. In the case of introduction through an ill traveller originating from a West African country where the outbreak is ongoing, it is important to strengthen the screening protocol for all incoming air passengers, rapidly detect and promptly isolate any ill traveller, confirm by laboratory diagnosis quickly and undertake rapid containment measures. In the case of an apparently healthy individual presenting to a health facility with unexplained symptoms of fever, profound weakness and diarrhoea, which are some of the early nonspecific symptoms of EVD, it would be essential for the clinician to obtain a careful and prompt travel history if the person had arrived from West Africa in the past 21 days (maximum incubation period of the disease). Without a proper travel history, cases may not be recognized early because haemorrhagic complications occur in fewer than half of those infected with Ebola virus and gross bleeding is relatively rare $(15,16)$.

Identifying infected individuals quickly requires accessible diagnostic and treatment facilities. The greatest risk of transmission of Ebola virus is not from patients with diagnosed infection but from delayed detection and isolation. Since the early symptoms of EVD are non-specific, patients with EVD may expose family caregivers and health-care workers before the infection is diagnosed (6). A high index of suspicion early on, proper isolation of a suspected patient, rapid laboratory confirmation, appropriate infection-control practices and epidemiological investigations should quickly limit the spread of the virus should it be introduced in the Region.

\section{Control measures for preventing transmission and amplification}

In the absence of any drug or vaccine of proven efficacy, the control measures for the EVD should be directed towards prevention of spread and containment. In all previous outbreaks, three core interventions were proved to have been effective in halting transmission (5). Because Ebola virus is spread mainly through direct contact with infected body fluids of symptomatic patients, usually faeces, vomit or blood (2), transmission can be stopped by a combination of: early diagnosis, surveillance and exhaustive contact tracing, and optimum patient care including isolation and effective infection control measures.

Once Ebola virus is suspected, early isolation of the patient and correct infection control measures are critical to prevent amplification. The need for this is highlighted by the fact that in all outbreaks of EVB, including the current one, it has been observed that specific conditions in hospitals and communities may facilitate the spread of the disease from human to human (12).

In order to break the chain of transmission once an infected person is identified or recognized, the most effective public health measure is contact tracing and monitoring the health of each contact, especially their temperature, daily for 21 days post exposure. If any of these contacts develops fever, they would have to be immediately isolated, tested and if found positive, interviewed to identify their contacts whose health should be monitored for another 21 days. An essential element for contact 


\section{Box 1 Key public health actions for early detection and prevention of transmission of Ebola virus [Source: (14)]}

\section{Leadership and coordination}

- Set up an intersectoral coordination mechanism for information sharing, periodic risk assessment and managing the national and international operational response should cases occur.

- Provide regular advice to travellers going to Ebola-affected countries on effective public health measures for limiting exposure.

\section{Screening at points of entry as required under the International Health Regulations (IHR, 2005)}

- Increase awareness of points of entry officials on the risk of importation of Ebola virus disease (EVD) case.

- Reinforce the team managing the screening of passengers arriving at the points of entry by deploying additional human resources.

- Ensure that the points of entry officials are appropriately trained for assessment, screening and detection of any suspected case with EVD.

- Ensure availability of measures for isolation of suspected case (possibly in a separate isolation/quarantine space) for investigation and management.

- Ensure there is effective coordination for information sharing and reporting between the designated points of entry officials and the Ministry of Health.

\section{Enhancing surveillance for contact tracing and monitoring}

- Enhance surveillance for clusters of unexplained deaths or febrile illness in countries with links to ground border crossing.

- Disseminate case definition of EVD to all health care facilities.

- Develop a protocol for active surveillance, contact tracing and monitoring of close contacts of any suspected case detected or identified either at the points of entry or through the nation's disease surveillance system.

- Ensure that the relevant staff are trained on field epidemiology, investigation and data management.

\section{Reinforcing infection control precautions in health-care settings}

- Identify, designate and establish (where necessary) appropriate isolation units for patient care.

- Ensure availability of sufficient quantities of personal protective equipment (PPEs) and other disinfectant materials at point of care.

- Ensure that the health-care workers are appropriately trained on the use, especially on properly putting on and removing PPEs and other barrier nursing practices for care of EVD patient in health care facilities.

\section{Improving access to quality diagnostics testing and rapid turnaround of results}

- Train staff on collection, storage and transportation of samples from suspected cases and deactivation of specimens ensuring appropriate biosecurity measures.

- Establish a process for rapid shipment of specimens to a WHO reference laboratory.

- Ensure capacity to perform routine diagnostic testing.

\section{Improving social mobilization and risk communication for Ebola}

- Provide essential information on EVD to general and at-risk populations (health-care workers, for example).

- Advise identified target audiences (health-care workers and family members) on what can be done to prevent the virus from spreading.

- Develop key messages on preparedness and modify as the situation evolves

- Develop communications products for various target groups.

- Ensure capacity to perform social mobilization and risk communication if and when an outbreak occurs. 
tracing is to engage the community. Culturally acceptable risk communication messages that can overcome ignorance, allay fears and establish trust between the community and the public health officials have been found to be effective for successful case identification and contact tracing. Involving religious leaders can also be beneficial.

At the same time, aggressive supportive care should be given to a patient with Ebola. Although clinical data remain few, it seems likely that effective basic supportive care may make the difference between life and death for an infected patient (4).

It is essential to recognize that preparedness and response measures cannot be properly implemented without adequate numbers of trained staff assigned for screening at the points of entry, rigorous case identification, surveillance and care of patients and primary contacts, strict isolation of patients and good and supportive clinical care. Without sufficient human resources, prevention and containment measures are likely to be jeopardized.

EVD is not a mysterious disease. The proven interventions are straightforward. However, if a single case is missed, a single contact becomes ill and isn't isolated or if there is a single lapse in infection control, another chain of transmission would start and the disease may spiral out of control.

\section{Moving forward}

Despite the scale of the current outbreak of EVD, the degree of transmissibility of this virus seems to be similar to those seen in other epidemics. The particular fury with which this latest outbreak is raging cannot be attributed to the biological characteristics of the virus. The strain presently circulating in West Africa has 97\% homology to Zaire ebolavirus samples found in the Democratic Republic of Congo and Gabon (17). This strain has historically resulted in the highest mortality (90\%), although the estimated case fatality rate in the current outbreak is less than 60\% (5).

A combination of factors such as disintegrated or fragile health systems, shortages of health-care workers, constant movement of people between the porous border and the fact that the outbreak has spread to cities has made tracing of contacts and hence containment of the outbreak extremely challenging.

A number of countries in the Region are themselves facing complex emergencies with massive population displacement taking place. Within these difficult circumstances, the countries need to enhance their preparedness efforts for a prompt and rapid response to any sign of the introduction of the virus in order to contain it before it becomes widespread and established.

The experience in some countries of the Region in managing outbreaks from other infectious disease that have emerged recently, such as the Middle East respiratory syndrome coronavirus (MERS-CoV) and pandemic influenza (H1N1), resulted in these countries stepping up their preparedness measures. As a result they have already invested efforts to enhance their surveillance, improve laboratory diagnostic and risk communication capacity and strengthen infection control practices in health facilities. The Ebola threat should motivate all countries to further improve their public health preparedness measures in all these areas.

The threat Ebola virus poses should not be taken lightly; it is no ordinary infection. Unless proper infection control measures are prepared and ready, it can challenge the health systems and undermine our ability to contain its rapid spread in hospitals and households. Furthermore, there could be political, economic and social disruptions and ramifications associated with an Ebola outbreak. Therefore, our response to the current threat of Ebola virus needs to be effective and decisive. Ebola is not the first, nor will it be the last, health danger to emerge that can threaten the Region. It remains a moral imperative for each and every country in the Region to have systems in place that can identify, stop and prevent this and any future threats to the health of their populations and their national security.

\section{References}

1. Briand S, Bertherat E, Cox P, Formenty P, Kieny MP, Myhre JK, et al. The international Ebola emergency. N Engl J Med. 2014 Sep 25;371(13):1180-3. 10.1056/NEJMp1409858 PMID:25140855

2. WHO Ebola Response Team. Ebola virus disease in West Africa - the first 9 Months of the epidemic and forward projections. N Engl J Med. 2014 Sep 22. [Epub ahead of print]. PMID:25244186

3. World Health Organization. WHO statement on the meeting of the International Health Regulations Emergency Committee regarding the 2014 the Ebola outbreak in West Africa [WHO statement 8 August 2014] (http://www.who.int/mediacentre/news/statements/2014/ebola-20140808/en/, accessed 30 September 2014).

4. Baden LR, Kanapathipillai R, Campion EW, Morrissey S, Rubin EJ, Drazen JM. Ebola - An Ongoing Crisis. N Engl J Med. 2014 Sep 19; 10.1056/NEJMe1411378 PMID:25237780
5. Fauci AS. Ebola-underscoring the global disparities in health care resources. N Engl J Med. 2014 Sep 18;371(12):1084-6. PMID:25119491

6. Frieden TR, Damon I, Bell BP, Kenyon T, Nichol S. Ebola 2014-new challenges, new global response and responsibility. N Engl J Med. 2014 Sep 25;371(13):1177-80. 10.1056/NEJMp1409903 PMID:25140858

7. WHO: Ebola Response Roadmap Situation Report. 8 October 2014. Geneva: World Health Organization (http://apps.who.int/iris/bitstream/10665/136020/1/ roadmapsitrep_8Oct2014_eng.pdf?ua=1, accessed 9 October 2014).

8. Ebola haemorrhagic fever in Sudan, 1976. Report of a WHO/International Study Team. Bull World Health Organ. 1978;56(2):247-70. PMID:307455 
9. WHO. Outbreak of Ebola haemorrhagic fever in Yambio, south Sudan, April-June 2004. Wkly Epidemiol Rec. 2005 Oct 28;80(43):370-5. PMID:16285261

10. Farrar JJ, Piot P. The Ebola Emergency - Immediate Action, Ongoing Strategy. N Engl J Med. 2014 Sep 22; 10.1056/NEJMe1411471 PMID:25244185

11. Gomes MFC, P.y.P.A., Rossi L, Chao D, Longini I, Halloran ME, Vespignani A. Assessing the International Spreading Risk Associated with the 2014 West African Ebola Outbreak. PLOS Currents Outbreaks. 2014 Sep 2. Edition 1.

12. Breman JG, Johnson KM. Ebola Then and Now. N Engl J Med. 2014 Sep 10; 10.1056/NEJMp1410540 PMID:25207624

13. Jahrling PB, Geisbert TW, Dalgard DW, Johnson ED, Ksiazek TG, Hall WC, et al. Preliminary report: isolation of Ebola virus from monkeys imported to USA. Lancet. $1990 \mathrm{Mar}$ 3;335(8688):502-5. PMID: 1968529
14. Ebola and Marburg virus disease epidemics: preparedness, alert, control, and evaluation. Interim version 1.2, August 2014. Geneva: World Health Organization; 2014 (http:// apps.who.int/iris/bitstream/10665/130160/1/WHO_HSE_ PED_CED_2014.05_eng.pdf?ua=1\&ua=1, accessed 8 October 2014).

15. Feldmann H, Geisbert TW. Ebola haemorrhagic fever. Lancet. 2011 Mar 5;377(9768):849-62. PMID: 21084112

16. Bwaka MA, Bonnet MJ, Calain P. Ebola hemorrhagic fever in Kikwit, Democratic Republic of Congo: clinical observations in 103 patients. J Infect Dis. 1999 Feb;179 Suppl 1:S1-7. PMID: 9988155

17. Baize S, Pannetier D, Oestereich L, Rieger T, Koivogui L, Magassouba N, et al. Emergence of Zaire Ebola Virus Disease in Guinea. N Engl J Med. 2014 Apr 16; 10.1056/NEJMoa1404505 PMID:24738640 